\title{
Optimized Message Passing Schedules for LDPC Decoding
}

\author{
Predrag Radosavljevic, Alexandre de Baynast, and Joseph R. Cavallaro \\ Department of Electrical and Computer Engineering \\ Rice University, MS-366 - 6100 Main Street \\ Houston, Texas 77005 \\ \{rpredrag, debaynas, cavallar\}@ rice.edu
}

\begin{abstract}
The major drawback of the LDPC codes versus the turbo-codes is their comparative low convergence speed: $25-30$ iterations vs. 8-10 iterations for turbo-codes. Recently, Hocevar showed by simulations that the convergence rate of the LDPC decoder can be accelerated by exploiting a 'turbo-scheduling' applied on the bit-node messages (rows of the parity check matrix). In this paper, we show analytically that the convergence rate for this type of scheduling is about two times increased for most of the regular LDPC codes. Second we prove that 'turbo-scheduling' applied on the rows of the parity check matrix is identical belief propagation algorithm as standard message passing algorithm. Furthermore, we propose two new message passing schedules: 1) a turbo-scheduling is applied on the checknode messages (columns of the parity check matrix); 2) a hybrid version of both previous schedules where the turbo-effect is applied on both check-nodes and bit-nodes. Frame error rate simulations validate the effectiveness of the proposed schedules.
\end{abstract}

\section{INTRODUCTION}

${ }^{1}$ The low-density parity-check (LDPC) codes [1] are commonly decoded by using iterative standard message-passing (SMP) [2] algorithm shown on Fig. 1-top. About 25-30 iterations are necessary in order to match the performance of a turbo-decoder which requires only 8-10 iterations to converge [3]. Therefore, increasing the convergence speed of the LDPC decoder is essential. A 'turbo-scheduling' has been introduced in [4]. In [5] and [6] simulations show that the decoding convergence, if this algorithm is applied, is twice faster because of the better scheduling of messages.

The main idea is to better utilize the updated reliability messages within the single iteration. By exploiting how messages are scheduled during the decoding, it is possible to analytically predict convergence for different message-scheduling schemes, as well as the convergence speed-up (acceleration rate) comparing to the standard scheduling algorithm.

This paper is organized as follows. First we introduce three optimized message passing algorithms: row message passing (RMP), column message passing (CMP), and row-column message passing (RCMP) scheduling algorithm. In the third section RMP algorithm is closely analyzed and it is shown that both RMP and standard message passing (SMP) algorithm represent just a variation of the same belief propagation (BP)

\footnotetext{
${ }^{1}$ This work was supported in part by Nokia Corporation and by NSF under grants EIA-0224458 and EIA-0321266.
}

algorithm. In the fourth section we show analytically that the convergence rate of LDPC decoding algorithm based on RMP scheduling is about two times increased for most of the regular LDPC codes comparing to SMP scheduling. Finally, we show by frame error rate simulations that the convergence rate of these three schedules is approximatively twice as fast as SMP for irregular codes as well.

\section{Four Different Message Passing Schedules}

The LDPC codes can be represented by a bipartite graph that consists of two types of nodes: the bit-nodes and the checknodes [2]. Each coded bit is represented by a bit-node, whereas each parity check equation represents a check-node. The BP algorithm is used to decode the LDPC codes iteratively [2]. Bit-nodes and check-nodes exchange messages according to a pre-determined schedule. In this section, we successively describe four different schedules.

\section{A. Standard Message Passing (SMP) Schedule}

The standard message passing schedule is a two-phase iterative schedule (phases $P_{1}, P_{2}$ ) as shown on Fig. 1 . We define the Psi-function as in [5]: $\Psi(x)=-\log \left[\tanh \left(\frac{|x|}{2}\right)\right]$. $N(m)$ and $M(j)$ denote the subsets of the column indices corresponding to the location of the $1 \mathrm{~s}$ in the row $m$ and the column $j$ of the PCM, respectively. The channel log-likelihood ratios $L L R_{j}$ related to the bit nodes $j, j=1, \ldots, N$ are equal to $2 r_{j} / \sigma^{2}$ where $r_{j}$ is the $j$-th noisy received sample, $j=1, \ldots, N$ and $\sigma^{2}$ is the noise variance of the channel. Through the whole paper, we assume an AWGN transmission channel. Thus, the decoding algorithm is [5]:

P1: do for every row $m$ (a): Update check-equation probability $R_{m}$ :

$$
R_{m}=\prod_{n \in N(m)} \operatorname{sgn}\left(L\left(q_{m n}\right)\right) \times \Psi\left(\sum_{n \in N(m)} \Psi\left(L\left(q_{m n}\right)\right)\right)
$$

(b): Updating of the check-node messages $\forall j$ :

$R_{m j}=\operatorname{sgn}\left(R_{m}\right) \times \operatorname{sgn}\left(L q_{m j}\right) \times \Psi\left[\Psi\left(R_{m}\right)-\Psi\left(L\left(q_{m j}\right)\right)\right]$

P2: do for every column $j$ 


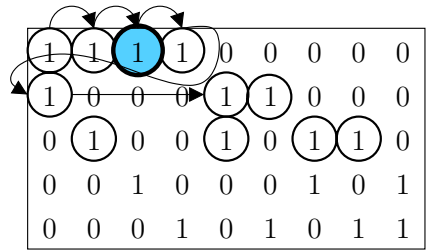

Phase $1\left(R_{m j}\right)$

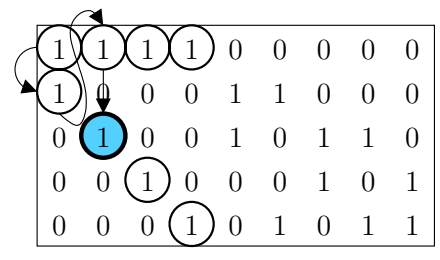

Phase $2\left(L\left(q_{m j}\right)\right)$

\begin{tabular}{|lllllllll|}
\hline & 1 & 1 & 1 & 0 & 0 & 0 & 0 & 0 \\
\hline 1 & 0 & 0 & 0 & 1 & 1 & 0 & 0 & 0 \\
\hline 0 & 1 & 0 & 0 & 1 & 0 & 1 & 1 & 0 \\
\hline 0 & 0 & 1 & 0 & 0 & 0 & 1 & 0 & 1 \\
\hline 0 & 0 & 0 & 1 & 0 & 1 & 0 & 1 & 1 \\
\hline
\end{tabular}

RMP

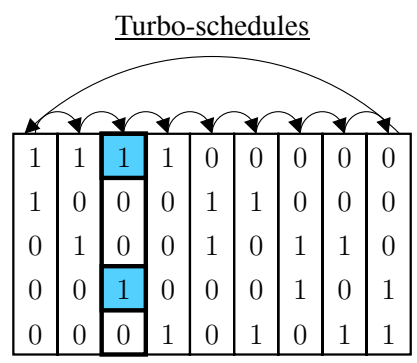

CMP

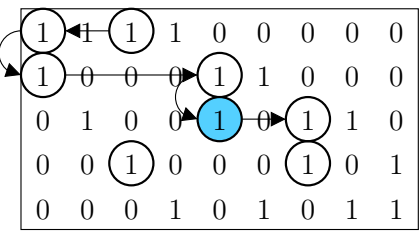

RCMP

Fig. 1. Four Message Passing Schedules for the LDPC iterative decoding algorithm - from top to bottom: Standard Message Passing (SMP): in the first phase all check-node probabilities are updated, then in the second phase all bit-node probabilities are updated; Row Message Passing (RMP): bit-node probabilities and check-node probabilities are updated row by row; Column Message Passing (CMP): bit-node probabilities and check-node probabilities are updated column by column; Row-Column Message Passing (RCMP): bit-node probabilities and check-node probabilities are updated single element by single element through random walk. The starting position is arbitrary, 1st row and 3rd column in this example.

(a): Updating of all a posteriori probability (APP) messages:

$$
L\left(q_{j}\right)=\sum_{m \in M(j)} R_{m j}+\operatorname{LLR}_{j}
$$

(b): Updating of the bit-node messages $\forall m$ :

$$
L\left(q_{m j}\right)=L\left(q_{j}\right)-R_{m j}
$$

Tentative decoding: Stop if all parity-check equations are verified or if a maximum number of iterations is reached. Otherwise, go to the step P1 (next decoding iteration).

We can summarize this message-passing schedule in the following compact form:

$$
\begin{gathered}
\{\forall m:(1) \rightarrow\{\forall j:(2)\}\} \rightarrow\{\forall j:(3) \rightarrow\{\forall m:(4)\}\} \\
\text { SMP schedule }
\end{gathered}
$$

\section{B. Row Message Passing (RMP) Schedule}

In [5], and [6], an improved message passing schedule is presented. It is shown by simulations that the decoding process based on RMP converges about two times faster than the SMP schedule. We refer to it as the 'Row Message Passing' (RMP) schedule since the turbo-effect is applied on the rows of the PCM as shown on Fig. 1. For a given row $m, m=1, \ldots, M$, simultaneous updating of all reliability messages $R_{m j}, L\left(q_{j}\right)$ and $L\left(q_{m j}\right), j=1, \ldots, N$ is performed and the processing is repeated for all rows:

$$
\begin{aligned}
\forall m:\{\forall j:(4)\} & \rightarrow(1) \rightarrow\{\forall j:(2)\} \rightarrow\{\forall j:(3)\} \\
& \text { RMP schedule }
\end{aligned}
$$

In order to keep same computational complexity as in SMP, (3) can be simplified as follows:

$$
L\left(q_{j}\right)=R_{m j}+L\left(q_{m j}\right)
$$

It will be shown in section III that after some basic mathematical manipulations, the updating rule for APP messages given by (5) is equivalent to the expression defined by (3). However, faster convergence of RMP schedule is achieved by performing the partial summations of check messages.

\section{Column Message Passing (CMP) Schedule}

As for RMP, the turbo message passing effect can be also achieved by updating messages from column to column as shown on Fig. 1. For all check-nodes that participate in the current column $j$, simultaneous updating of all messages per one column is performed and this process is repeated for all columns:

$$
\begin{aligned}
\forall j:\{\forall m:(2)\} \rightarrow & (3) \rightarrow\{\forall m:(4)\} \rightarrow\{\forall m:(1)\} \\
& \text { CMP schedule }
\end{aligned}
$$


In order to keep the same computational complexity per iteration as for SMP, we can simplify (1) as follows:

$$
R_{m}=\operatorname{sgn}\left(R_{m j}\right) \operatorname{sgn}\left(L q_{m j}\right) \Psi\left[\Psi\left(R_{m j}\right)+\Psi\left(L\left(q_{m j}\right)\right)\right]
$$

\section{Row-Column Message Passing (RCMP) Schedule}

By combining RMP and CMP schedules it is possible to exploit a partial turbo message passing effect in two directions simultaneously (per row and per column) as shown on Fig. 1. This message scheduling is called Row-Column Message Passing (RCMP) schedule. For each coded bit $j$ in the parity check equation $m$ the following decoding steps are executed:

$$
\begin{aligned}
\forall m, j: & (4) \rightarrow(2) \rightarrow(1) \rightarrow(3) \\
& \text { RCMP schedule }
\end{aligned}
$$

Further simplifications can be applied by replacing (1) and (3) by (6) and (5), respectively.

\section{RMP ALGORITHM: OPTIMIZED SCHEDULING OF MESSAGES}

In this section the RMP scheduling algorithm is analyzed in more details. We show that both RMP and SMP schedules represent identical belief propagation algorithm. Typically about two times faster convergence of RMP is justified by showing that the scheduling of reliability messages is better in RMP than in SMP decoding scheme.

We first introduce the notion of layer in the PCM as in [5]: layer is a set of rows of PCM that can be processed independently (in parallel) without any performance loss. In each layer there is up to one non-zero entry per column. The analysis of RMP schedule does not depend on the size of layer nor on the number of layers: ultimately, the layer can be only one row of PCM. It is assumed that the total number of layers in the PCM is $L$ (the $L$-th layer is also the last layer of the PCM), there are $i$ decoding iterations, and $W_{R}$ is the connectivity degree of the check node.

The updating of bit-node probabilities in the last layer $L$ during the $i$-th iteration can be written according to (4) as:

$$
L\left(q_{m j}^{(L, i)}\right)=L\left(q_{j}^{(L-1, i)}\right)-R_{m j}^{(L, i-1)},
$$

where $L\left(q_{j}^{(L-1, i)}\right)$ represents APP message updated in the previous layer during the same $i$-th decoding iteration. It is assumed that all reliability messages are updated in every layer, which is not necessary condition for the analysis of RMP schedule.

The updating of check-node probabilities for the row $m$ which is the last one in column $j$ with non-zero entry, is a function of $W_{R^{-1}}$ previously updated bit-node probabilities that belong to the same row $m$ :

$$
R_{m j}^{(L, i)}=F\left(L\left(q_{m j 1}^{(L, i)}\right), \ldots, L\left(q_{m j\left(W_{R}-1\right)}^{(L, i)}\right)\right)
$$

The updating rule for APP messages given by (5) at the end of $i$-th decoding iteration can be rewritten as:

$$
\begin{aligned}
L\left(q_{j}^{(L, i)}\right) & =L\left(q_{m j}^{(L, i)}\right)+R_{m j}^{(L, i)} \\
& =L\left(q_{j}^{(L-1, i)}\right)+R_{m j}^{(L, i)}-R_{m j}^{(L, i-1)}
\end{aligned}
$$

Equation 9 can be extended for all previous $L$-1 layers in the $i$-th decoding iteration:

$$
\begin{aligned}
L\left(q_{j}^{(L-1, i)}\right) & =L\left(q_{j}^{(L-2, i)}\right)+R_{m j}^{(L-1, i)}-R_{m j}^{(L-1, i-1)} \\
& \vdots \\
L\left(q_{j}^{(1, i)}\right) & =L\left(q_{j}^{(0, i)}\right)+R_{m j}^{(1, i)}-R_{m j}^{(1, i-1)} \\
& =L\left(q_{j}^{(L, i-1)}\right)+R_{m j}^{(1, i)}-R_{m j}^{(1, i-1)}
\end{aligned}
$$

After adding all previous equations that correspond to the updating of APP messages in column $j$ it follows that:

$$
L\left(q_{j}^{(L, i)}\right)=L\left(q_{j}^{(L, i-1)}\right)+\sum_{m \in M(j)} R_{m j}^{(i)}-\sum_{m \in M(j)} R_{m j}^{(i-1)}
$$

Equation 11 can be extended for all previous $i$-1 decoding iterations:

$$
\begin{aligned}
L\left(q_{j}^{(L, i-1)}\right) & =L\left(q_{j}^{(L, i-2)}\right)+\sum_{m \in M(j)} R_{m j}^{(i-1)}-\sum_{m \in M(j)} R_{m j}^{(i-2)} \\
& \vdots \\
L\left(q_{j}^{(L, 1)}\right) & =L\left(q_{j}^{(L, 0)}\right)+\sum_{m \in M(j)} R_{m j}^{(1)}-\sum_{m \in M(j)} R_{m j}^{(0)}
\end{aligned}
$$

The APP messages are initialized with the channel LLRs at the beginning of the first iteration, where $N$ is the total number of transmitted bits.

$$
L\left(q_{j}^{(L, 0)}\right)=L\left(q_{j}^{(1,1)}\right)=\frac{2 r_{j}}{\sigma^{2}}, \quad \forall j=1, \ldots, N
$$

All check-messages are initialized with zero at the beginning of the first decoding iteration, where $K$ is the number of transmitted information bits:

$$
R_{m j}^{(0)}=0, \quad \forall m=1, \ldots, N-K ; \quad \forall j=1, \ldots, N
$$

After adding (11) through (12) ( $i$ equations for all decoding iterations) it follows that APP message for the bit-node $j$ at the end of $i$-th decoding iteration is given by:

$$
\begin{aligned}
L\left(q_{j}^{(L, i)}\right) & =L\left(q_{j}^{(L, 0)}\right)+\sum_{m \in M(j)} R_{m j}^{(i)}-\sum_{m \in M(j)} R_{m j}^{(0)} \\
& =\frac{2 r_{j}}{\sigma^{2}}+\sum_{m \in M(j)} R_{m j}^{(i)}
\end{aligned}
$$

From (15) (updating rule for APP messages in RMP schedule) and (3) (updating rule for APP messages in SMP schedule), we conclude that the final mathematical expressions for updating of APP messages are identical for two different scheduling schemes and therefore both algorithms represent the same approximation of the belief propagation algorithm. The only difference and the main advantage of RMP algorithm is optimized scheduling of reliability messages that causes faster 
decoding convergence. After combining (7), and (11) for any layer $L_{m}\left(L_{m}=1, \ldots, L\right.$, where row $m$ belongs to the layer $L_{m}$ ) and iteration $i$, any bit-node message inside this layer can be computed as:

$$
\begin{aligned}
L\left(q_{m j}^{(i)}\right) & =L\left(q_{j}^{(i-1)}\right)-\sum_{l=1}^{L_{m}-1} R_{l j}^{(i-1)}+\sum_{l=1}^{L_{m}-1} R_{l j}^{(i)}-R_{m j}^{(i-1)} \\
& =L\left(q_{j}^{(i-1)}\right)-\sum_{l=1}^{L_{m}} R_{l j}^{(i-1)}+\sum_{l=1}^{L_{m}-1} R_{l j}^{(i)},
\end{aligned}
$$

where $L\left(q_{j}^{(i-1)}\right)$ is APP message updated at the end of iteration $i$-1. From (15) applied for the decoding iteration $i-1$ and (16) it follows that:

$$
L\left(q_{m j}^{(i)}\right)=\frac{2 r_{j}}{\sigma^{2}}+\sum_{l=1}^{L_{m}-1} R_{l j}^{(i)}+\sum_{l=L_{m}+1}^{L} R_{l j}^{(i-1)}
$$

In the same time by combining (3) and (4), the updating of the bit-node message from layer $L_{m}$ for the SMP schedule is given by:

$$
L\left(q_{m j}^{(i)}\right)=\frac{2 r_{j}}{\sigma^{2}}+\sum_{l=1, l \neq L_{m}}^{L} R_{l j}^{(i-1)}
$$

After comparing (17) and (18) it is important to note that in the RMP algorithm check-messages updated during the current decoding iteration are immediately utilized within the same iteration for computation of new bit-node messages. In SMP algorithm only check messages from previous iteration are used in updating of bit-node messages. Thus, the reliability of bit-node messages improves faster using RMP than SMP scheduling scheme. Consequently, the reliability of check messages and APP messages are also better and overall decoding convergence of RMP schedule is faster.

\section{Convergence Analysis}

In this section we compare the convergence speed of RMP and SMP scheduling schemes. Several solutions for the estimation of the convergence acceleration rate have been recently proposed. In [7], the convergence acceleration rate is derived using Density Evolution Analysis (DEA). The result is very accurate, but the proposed solution is not analytical (obtained through the simulations). In [8], estimation of the convergence factor $\alpha$ is based on the number of channel LLRs used for the computation of one APP message at the end of $i$-th decoding iteration. However, this upper bound is not tight especially for large check-node connectivity degree. In this paper we introduce a new metric that provides us a more accurate analytical approximation of the convergence speed: we count the number of check-messages updated during the decoding iteration $i$-1 involved in the computation of one APP message $L\left(q_{j}\right)$ at the end of $i$-th decoding iteration.

We assume (only for the sake of computational simplicity) regular Gallager codes [4] with no cycles. Gallager codes have non-zero entry in every column per layer: if bit-node connectivity degree is $W_{c}$, there are $W_{c}$ layers in the PCM (also there is a constant check-node connectivity degree of
$\left.W_{R}\right)$. Let us denote the number of updated check messages from iteration $i$-1 utilized in computation of one APP message at the end of next iteration $i$ as $N_{R M P}$ and $N_{S M P}$ for RMP and SMP scheduling algorithms, respectively. Convergence acceleration rate $\alpha$ represents how many times the number of decoding iterations needs to be increased in SMP schedule to reach the same number of utilized check messages in RMP schedule: number of check messages updated $\alpha i$ SMP iterations ago used in the computation of one APP message at the end of current iteration is equal to the number of utilized check messages that are updated $i$ RMP iterations ago. Consequently, the convergence factor $\alpha$ is defined with the following equality relation:

$$
N_{S M P}^{(\alpha i)}=N_{R M P}^{(i)}
$$

It can be shown that the number of check messages previously updated ( $i$ iterations ago) used in the computation of one APP message at the end of the current decoding iterations (after $i$ decoding iterations) is approximately polynomial function of iteration number and the number of updated check messages from previous iteration utilized in computation of one APP message at the end of next iteration:

$$
N_{S M P}^{(\alpha i)} \approx N_{S M P}^{\alpha i} ; \quad N_{R M P}^{(i)} \approx N_{R M P}^{i} ;
$$

After combining (19) and (20) the convergence rate in the case of RMP schedule $\left(\alpha_{R M P}\right)$ can be expressed as:

$$
\alpha_{R M P} \approx \frac{\log N_{R M P}^{i}}{\log N_{S M P}^{i}}=\frac{\log N_{R M P}}{\log N_{S M P}}
$$

According to (21), the convergence rate is defined as a log ratio between the numbers of check-messages updated in the previous decoding iteration used in the computation of one APP message at the end of current iteration for RMP and SMP schedules respectively. Numbers of utilized check messages from previous to next decoding iteration for SMP and RMP decoding algorithms are:

$$
\begin{gathered}
N_{S M P}=W_{C}\left(W_{C}-1\right)\left(W_{R}-1\right) \\
N_{R M P}=\frac{W_{R}^{W_{C}}-1}{W_{R}-1}\left[\left(W_{R}-1\right)\left(W_{C}-1\right)-1\right]+W_{C}
\end{gathered}
$$

In both cases $W_{C}$ check messages updated in the current iteration are used in the computation of one APP message. Equation 22 is derived by knowing that there are $\left(W_{C^{-}}-1\right)\left(W_{R^{-}}\right.$ 1) check messages from the previous iteration utilized in the computation of one check message from the current iteration. In RMP schedule, the number of utilized check messages from previous iteration $i-1$ for computation of one check message in iteration $i$ is determined by the recursive formula per layer $l\left(l=1, \ldots, L_{m}, \ldots, W_{C}\right)$ derived from (17):

$$
\left(W_{R}-1\right)\left[\left(W_{C}-L_{m}\right)+\sum_{l=1}^{L_{m}-1} \# R_{l j}^{(i)}\right] \text {, }
$$

where $\# R_{l j}^{(i)}$ represents the number of check messages from previous iteration $i$-1 utilized for the computation of one 
check message from the $l$-th layer in the current iteration $i$. It is known that check message $R_{1 j}^{(i)}$ (first layer of the $i$ th iteration) utilizes $\left(W_{C^{-}}-1\right)\left(W_{R}-1\right)$ check messages from the previous iteration $i$-1. After adding up results of the recursive formula (24) for all $W_{C}$ layers, the total number of utilized check messages from previous iteration is equal to (23).

The log ratio between (23) and (22) represents the RMP convergence accelerating factor $\alpha_{R M P}$ comparatively to the SMP schedule. Furthermore, by simulations shown in Fig. 2 we estimate the acceleration rate $\hat{\alpha}$ as the ratio between the average number of iterations required to reach the Frame Error Rate (FER) of approximatively $10^{-4}$ for SMP scheduling algorithm and any improved message passing (IMP) scheduling algorithms (RMP, CMP, and RCMP).

$$
\hat{\alpha}_{I M P}=\frac{(\text { Av.Iter })_{S M P}}{(\text { Av.Iter })_{I M P}}
$$

As shown in Fig. 2, the analytical convergence factor for the

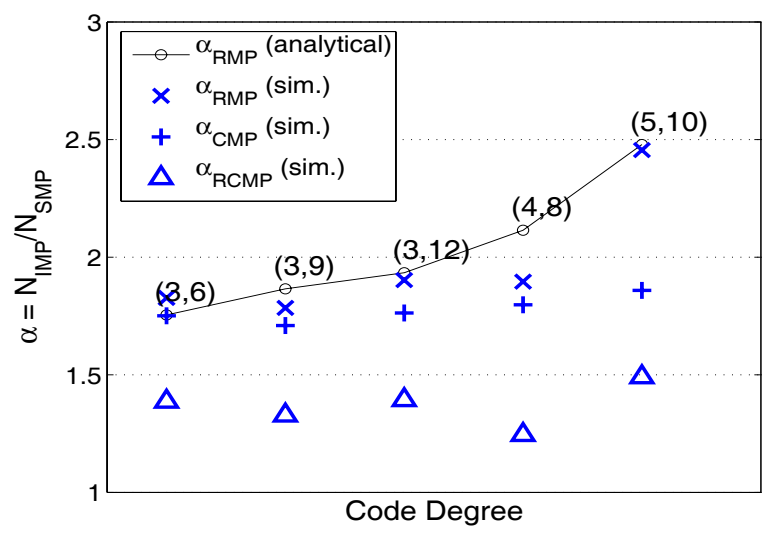

Fig. 2. Comparison of the convergence accelerating factor $\alpha$ defined in (19), (25) for the three improved schedules RMP, CMP, RCMP relatively to the SMP schedule. The convergence factor is determined for several regular codes $\left(W_{C}=3, W_{R}=6\right),(3,9),(3,12),(4,8)$ and $(5,10)$.

RMP schedule given in (21) coincides well with the simulated values for several regular codes. The convergence accelerating factor for the CMP schedule is slightly slower ([6]) comparing to RMP, while RCMP schedule has the slowest decoding convergence among the three proposed schedules. All three outperforms the SMP schedule.

\section{Simulation Results}

In this section, we show FER performance for irregular LDPC codes of moderate frame length $(N=3000$ bits $)$ compatible with the IEEE 802.16e standard [9]. The maximum number of iterations is fixed to 15 for RMP, CMP and RCMP schedules. For the SMP schedule, performance results for maximum number of iterations of 15 and 30 are presented. As expected from the convergence analysis given in Sec. IV, the convergence speed of RMP and CMP schedules is about twice faster than for SMP schedule. The performance results for RCMP schedule is similar to RMP and CMP, but the average number of iterations is significantly larger.

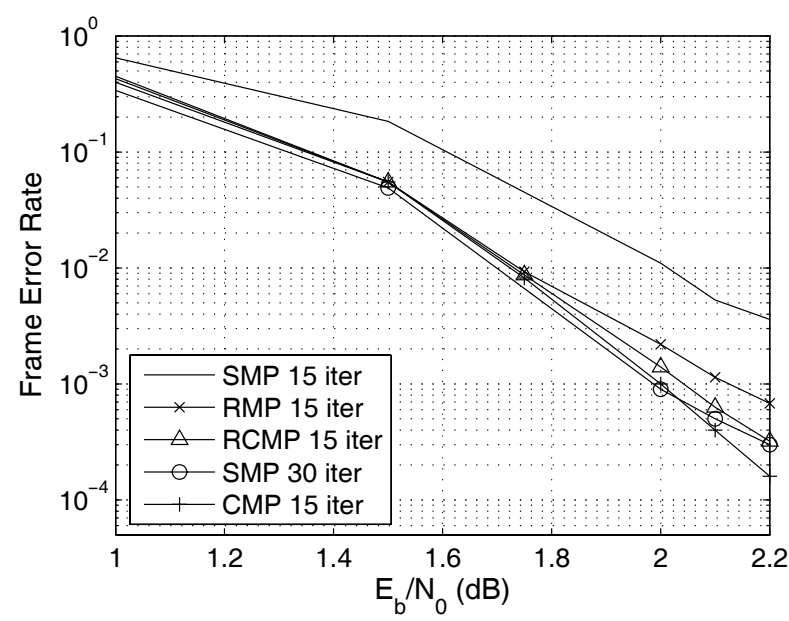

Fig. 3. Performance of the LDPC decoder over AWGN channel for the different Message-Passing schedules - SMP, RMP, CMP, RCMP. For RMP, CMP and RCMP schedules, the maximum number of iterations is set to 15; for SMP schedule, the results are shown for both 15 and 30 iterations.

\section{CONCLUSION}

We presented four variations of the belief propagation algorithm for LDPC decoding based on different scheduling of reliability messages. It is shown that RMP (CMP and RCMP as well) is the same belief propagation algorithm as SMP: identical computational complexity is achieved, the only difference that causes faster decoding convergence is optimized scheduling of messages. By introducing the convergence acceleration rate $\alpha$ for the RMP schedule, we are able to predict analytically the convergence speed-up for a variety of standard LDPC codes. Simulations validate our approach for regular and irregular codes.

\section{REFERENCES}

[1] R. Gallager, "Low-density parity-check codes," Information Theory, IEEE Transactions on, vol. 8, pp. 21-28, Jan. 1962.

[2] D. MacKay, "Good Error-Correcting Codes Based on Very Sparse Matrices," Information Theory, IEEE Transactions on, vol. 45, 1999.

[3] C. Berrou, A. Glavieux, and P. Thitimajshima, "Near optimum error correcting coding and decoding: Turbo-Codes," in Communications, 1993. Technical Program, Conference Record, IEEE International Conference on, May 1993.

[4] M. Mansour and N. Shanbhag, "High-throughput LDPC decoders," IEEE Tran. Very Large Scale Integration Systems, vol. 11, pp. 976 - 996, 2003.

[5] D. Hocevar, "A reduced complexity decoder architecture via layered decoding of LDPC codes," in Signal Processing Systems SIPS 2004. IEEE Workshop on, pp. 107 - 112, Oct. 2004.

[6] E. Sharon, S. Litsyn, and J. Goldberger, "An efficient message-passing schedule for LDPC decoding," in 23rd IEEE Convention of Electrical and Electronics Engineers in Israel, pp. 223 - 226, September 2004.

[7] A. de Baynast, P. Radosavljevic, J. Cavallaro, and A. Sabharwal, "On Turbo-Schedules for LDPC Decoding." submitted to IEEE Communications Letters, November 2005.

[8] A. de Baynast, P. Radosavljevic, J. Cavallaro, and A. Sabharwal, "TurboShedule for LDPC decoding," in 43-rd Annual Allerton Conference on Communication, Control and Computing, September 2005.

[9] V. Stolpman et al., "LDPC coding for OFDMA PHY," Tech. Rep. IEEE C802.16e-04/526, IEEE 802.16 Broadband Wireless Access Working Group, 2004. 